\title{
PRÉSENTATION ET ESSAI D'UN COMPTEUR A RÉSISTIVITÉ POUR POISSONS
}

\author{
C. GOSSET
}

\author{
I.N.R.A. - Laboratoire d'Écologie des Poissons \\ Station d'Hydrobiologie \\ Saint-Pée-sur-Nivelle - 64310 ASCAIN (France)
}

Recu le 25 juin 1986

Accepté le 13 octobre 1986

Received 25 June 1986 Accepted 13 October 1986

\section{RESUME}

Un compteur à résistivité pour poissons, de fabrication récente, a été installé et testé dans la passe à poissons d'Uxondoa située sur la Basse Nivelle, dans les Pyrénées-Atlantiques.

Le fonctionnement de l'appareil électronique de comptage et les détails de construction et d'installation du détecteur à tunnel sont présentés dans cet article.

Les résultats des comptages effectués en 1985 sont exposés et analysés. Ils montrent, en particulier, que le fonctionnement est satisfaisant mais que la précision doit être sensiblement améliorée.

\section{DESCRIPTION AND TEST OF A RESISTIVITY FISH COUNTER}

\section{ABSTRACT}

A recently designed resistivity fish counter has been installed and tested at Uxondoa fish pass located in the lower Nivelle, in the Pyrénées-Atlantiques.

The functioning of the electronic counter and details on the tunnel detector set up are presented in this paper.

Results of counts made in 1985 are analysed. They show, in particular, that the apparatus works satisfactorily but that its accuracy has to be appreciably improved.

\section{INTRODUCTION}

Des études et des actions conduites par l'INRA pour la restauration du peuplement de saumons (Salmo salar) de la Nivelle, petit fleuve côtier des Pyrénées-Atlantiques, ont été entreprises en 1972. Ces travaux sont effectués en collaboration avec le Ministère de l'Environnement, l'Établissement Public Régional d'Aquitaine et la Fédération des Associations de Pêcheurs des Pyrénées-Atlantiques.

Le développement de l'un des principaux thèmes abordés: "Contrôle des stocks d'adultes sauvages et d'élevage" (DUMAS et CASAUBON, 1985) nécessite la capture des échantillons de pré-reproducteurs ou de reproducteurs. Jusqu'en 1983, ces captures étaient principalement effectuées par pêche électrique. Depuis 1984, ce travail a été grandement facilité et amélioré par la mise en service d'une passe à poissons et d'un piège de contrôle financés par la Fédération des AAPP des Pyrénées-Atlantiques et gérés par I'INRA.

La passe à poissons d'Uxondoa est constituée de 7 bassins successifs à échancrures latérales (LARINIER, 1983) et 'd'une chambre de capture. Le suivi de cette passe : structure de la population de saumons, rythmes de remontée (DUMAS, 1986), montre indubitablement son efficacité et son importance dans le contrôle des populations migratrices de salmonidés.

C'est dans cet ouvrage, lieu de passage obligatoire des salmonidés lors de leur migration amont - excepté quelques jours par an lors des crues importantes - que le Laboratoire d'Écologie des Poissons de la Station d'Hydrobiologie de l'INRA à St-Pée-sur-Nivelle a installé un compteur automatique à poissons. L'appareil électronique de comptage utilisé est commercialisé par les Établissements FRON à Toulouse. Ce compteur, conçu en collaboration avec le Centre National du Machinisme Agricole, du Génie Rural, des Eaux et Forêts (CEMAGREF, Antenne de Toulouse), est actuellement le seul appareil français disponible sur le marché.

\section{I - MATÉRIEL ET METHODES}

\section{1. - Les différents types de compteurs}

Rappelons brièvement qu'il existe plusieurs types de compteurs automatiques à poissons utilisant des principes fondamentalement différents: 
- Le compteur mécanique ou électromécanique est assez peu utilisé ou réservé à des usages particuliers. Le poisson se force un passage à travers un réseau de fils ou soulève des barreaux mobiles autour d'un axe horizontal, provoquant ainsi le déclenchement d'un système de comptage électromécanique ou électronique. La fiabilité de ce système peut, en particulier dans le cas du saumon lors de sa migration amont, compenser largement la gêne que le capteur peut occasionner aux animaux.

- Le compteur bioélectrique exploite les signaux électriques de faible amplitude induits par les contractions musculaires du poisson. Il est très peu utilisé car sa vulnérabilité aux interférences et bruits électriques le rend peu fiable. II ne permet pas non plus la détermination du sens de passage.

- Le compteur à cellules photoélectriques donne une information quand le faisceau lumineux est occulté par un poisson. Les principaux inconvénients de cette technique, turbidité de l'eau, obstruction des têtes de cellules par le développement d'algues et comptages erronés dus aux débris dérivants, semblent avoir été surmontés (BELL et ARMSTRONG, 1970). Cette technique est cependant peu utilisée.

- Le compteur à résistivité fonctionne grâce à lá différence de conductivité entre l'eau et le poisson. Les capteurs sont du type "tunnel" ou "canal ouvert". Dans le premier cas on oblige par un ouvrage associé le poisson à traverser un tube de forme circulaire ou prismatique équipé d'électrodes ; dans le second cas les électrodes sont disposées sur le fond de la rivière, dans le sens transversal, et fixées la plupart du temps sur le parement aval d'un seuil en béton. Le principal inconvénient du capteur "tunnel" est de nécessiter une infrastructure associée importante, tandis que la fiabilité des capteurs "canal ouvert" est très affectée par les variations de niveau de la rivière.

Ce type de compteur a surtout été étudié et développé par la "North of Scotland Hydro-electric Board" (NSHB) au Royaume-Uni depuis 1950 (BUSSELS, 1978), où un très grand nombre d'installations sont actuellement en service.

Des modèles à "tunnels multiples" plus sophistiqués mais d'une grande précision, mis au point par "Pulsar Electronics Ltd of Vancouver" et "Smith Boot Inc. of Vancouver" sont également utilisés, sur la côte ouest du Canada en particulier.

- Dans les compteurs "sonar" à ultrasons on émet des vibrations à fréquence ultrasonique dont les échos, renvoyés par le poisson, sont captés et analysés. L'émission et la réception sont assurées par des transformateurs d'énergie électrique en énergie mécanique appelés transducteurs. Ce type de compteur peut se révéler très performant et ne nécessite pas d'ouvrage associé important. C'est toutefois un système très sophistiqué et onéreux qui engendre quelques difficultés de mise en œuvre: choix du site délicat, exploitation très spécialisée, nettoyages et contrôles fréquents.

Cette technique semble avoir été abandonnée au Royaume-Uni mais est très développée par "Bendix Corporation" et "Biosonics Inc." aux U.S.A. Un modèle de compteur sonar à balayage de Bendix, largement utilisé sur la côte ouest du Canada, permet le comptage de plusieurs milliers de poissons par heure avec une précision supérieure à $95 \%$ (COUSENS et al., 1982). Des prototypes utilisant l'effet Doppler (variation de fréquence de l'écho réfléchi) sont également étudiés.

\section{2. - Le compteur à résistivité à tunnel}

\subsection{1. - Généralités}

Le compteur utilisé pour notre expérimentation se compose de 2 unités séparées: l'appareil électronique de comptage installé sur la berge dans un endroit abrité et le détecteur plongé dans l'eau (photo 1). Ce dernier est constitué par un tube isolant à l'intérieur duquel sont fixées les électrodes. Un câble à 3 conducteurs établit la liaison.

Le principe de mesure est bien connu : les deux impédances, constituées par l'eau entourant les trois électrodes régulièrement espacées, représentent deux branches d'un pont de Wheatstone équilibré en l'absence de poisson. La conductivité $\left({ }^{*}\right)$ de la chair du poisson ( $\gamma$ p) étant différente de la conductivité de l'eau ( $\gamma$ e), le passage d'un animal entre les électrodes provoque une variation de ces impédances et par conséquent un déséquilibre du pont de mesure. Le signal recueilli est ensuite amplifié et traité par une unité logique.

II apparaît clairement que le principe même de la mesure constitue en lui-même une limitation d'emploi. En effet, le signal de déséquilibre sera nul ou trop faible par rapport au bruit électrique ambiant pour être exploité, si la conductivité du poisson est égale ou peu différente de celle de l'eau.

(") Rappelons que : conductivité $(\mu \mathrm{S} / \mathrm{cm})=\frac{10^{6}}{\text { résistivité }(\Omega . \mathrm{cm})}$ 
Mais dans la majorité des cas, en eau douce en particulier, $\succ$ p est supérieur à $\succ$ e. Dans le cas de la Nivelle, par exemple, on a $\gamma e=250 \mu \mathrm{S} / \mathrm{cm}$, alors que nous avons mesuré par une méthode d'égalisation de conductivité eau-poisson (SHENTIAKOV, 1959) $\gamma p=2.000 \mu \mathrm{S} / \mathrm{cm}$ à $18^{\circ} \mathrm{C}$ (truite de mer).

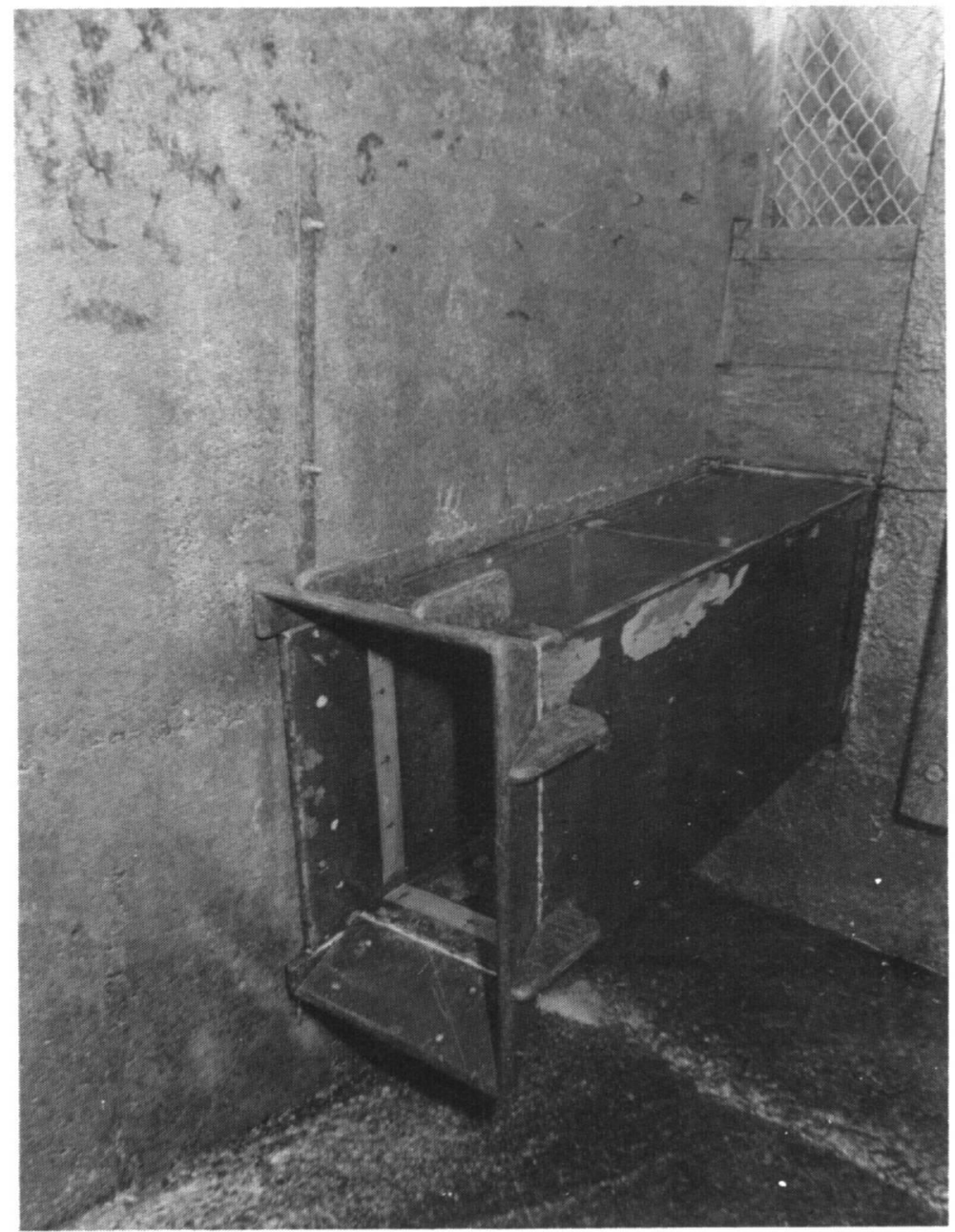

Photo 1 : Le tunnel détecteur dans la passe à poissons.

Photo 1 : The detector tunnel in the fish pass.

\subsection{2. - L'unité de comptage}

Le schéma synoptique du compteur FRON est donné à la figure 1. L'oscillateur basse fréquence délivre un signal d'horloge qui pilote un circuit bistable à $1 \mathrm{kHz}$. Après amplification de puissance, ce signal alimente en courant alternatif le pont de Wheatstone. L'équilibrage manuel du pont au repos est obtenu par un potentiomètre, et deux boutons poussoirs de test permettent de simuler le passage d'un poisson, en provoquant deux déséquilibres successifs et orientés. Le signal de déséquilibre recueilli est amplifié par un amplificateur à gain ajustable, et dirigé :

- d'une part vers les circuits de signalisation d'équilibre du pont (galvanomètre et diode LED);

- d'autre part vers l'entrée d'un circuit échantillonneur-bloqueur assurant la détection en synchronisme avec l'échantillonnage et donc la comparaison de phase qui permet d'obtenir l'indication du sens de passage. Les impulsions de synchronisation (ou d'horloge) sont prélevées sur une sortie décodée du compteur décimal. Le signal analogique est disponible en sortie de l'échantillonneur.

Le circuit de rattrapage de zéro permet de s'affranchir des variations lentes ou des déséquilibres permanents dus, par exemple, à la présence de débris entre les électrodes.

Après réinjection de la fréquence porteuse à $1 \mathrm{kHz}$, le signal résultant est traité par 4 comparateurs de tensions montés en parallèle qui, par l'intermédiaire de potentiomètres ajustables, déterminent :

- les seuils de commande du circuit de temporisation; 
- la discrimination des deux catégories de taille permettant l'aiguillage vers les compteurs électromagnétiques "petits + gros" (P) et "gros" (G); le compteur P compte tous les poissons qui provoquent un signai de déséquilibre supérieur à un seuil ajustable, le compteur $\mathrm{G}$ ne compte que les poissons qui induisent un signal supérieur à un autre seuil, plus élevé que le précédent. Le nombre des "petits" est donc obtenu par différence.

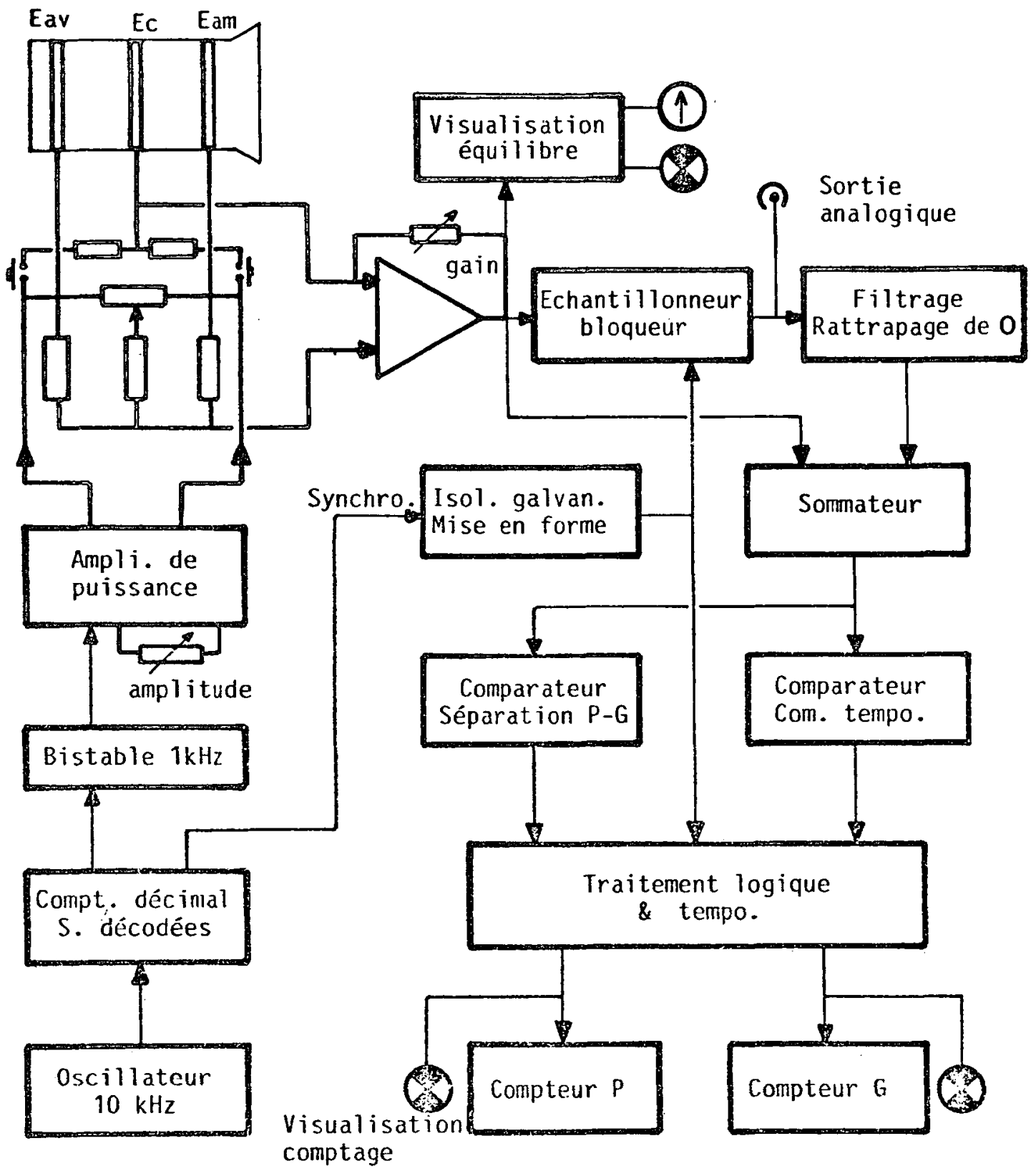

Figure 1 : Schéma synoptique du compteur électronique à poissons.

Figure 1 : Electronic fish counter - Block diagram.

La chaîne de traitement logique associée au comptage fonctionne également en synchronisme avec le signal d'alimentation du pont. Sa fonction est d'interdire le comptage dans deux cas :

- lorsqu'un délai supérieur à 6 secondes (porté à $10 \mathrm{sec}$ ) intervient entre le passage électrode aval (Eav) - électrode centrale (Ec) et Ec - électrode amont (Eam). On a ainsi voulu éliminer la possibilité de comptages multiples provoqués par des animaux ne traversant pas franchement le tunnel.

- Iorsqu'un passage amont-aval se produit, la séquence des déséquilibres étant alors inversée.

Les alimentations basse tension ( $\mp 15 \mathrm{~V}$ ) sont obtenues à l'aide de 2 convertisseurs de tension intégrés qui assurent également l'indépendance des masses du niveau du pont et des circuits de 
traitement du signal détecté. Cess convertisseurs sont alimentés soit à partir du secteur, soit à partir d'une batterie d'accumulateurs ( $24 \mathrm{~V}, 6 \mathrm{~A} . \mathrm{h}$ ) montée en tampon. Cette batterie, protégée contre les décharges profondes, assure l'alimentation du compteur en cas de pannes secteur de faible durée. Cette autonomie, variable suivant la conductivité de l'eau, est de l'ordre de 24 h dans le cas présent. Des voyants de signalisation (LED) permettent un contrôle rapide du bon fonctionnement des différents étages de l'alimentation.

\subsection{3. - Le tunnel détecteur}

Les caractéristiques principales du tunnel détecteur construit en contreplaqué marine de $2 \mathrm{~cm}$ d'épaisseur et équipé de 3 électrodes en acier inoxydable sont données à la figure 2. Le choix du plexiglas pour la construction de la partie supérieure a pour but de "sécuriser" le poisson en lui évitant d'emprunter un passage sombre. La forme et les dimensions de la section ont été choisies en fonction de la largeur des échancrures inter-bassins et de la hauteur d'eau moyenne.

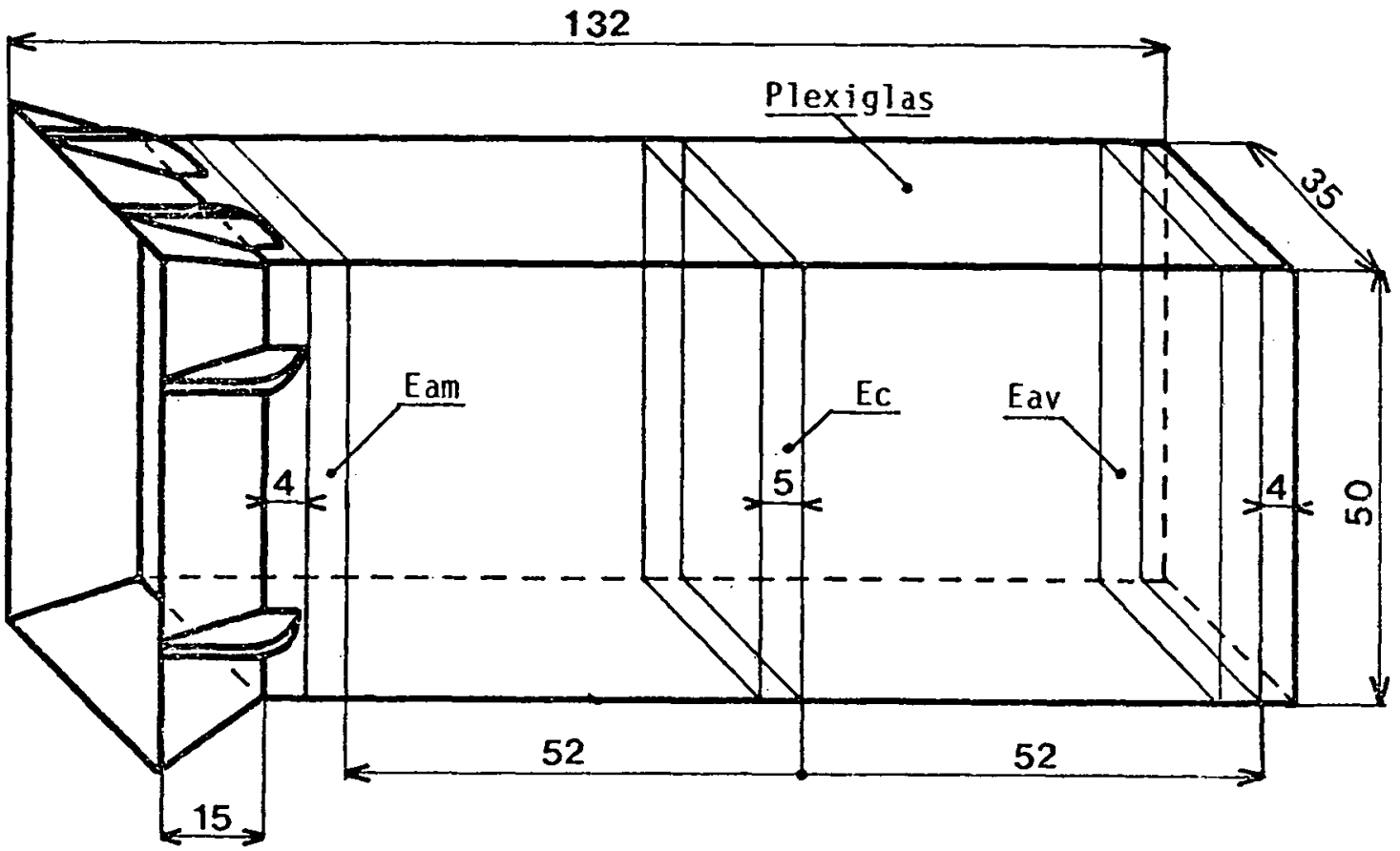

Figure 2 : Le tunnel détecteur

Figure 2 : The detector tunnel

La sensibilité maximale du compteur est obtenue lorsque la distance inter-électrodes est voisine de la longueur de la partie principale du corps de l'animal. L'écartement adopté résulte donc d'un compromis entre la longueur moyenne approximative des truites de $\operatorname{mer}(40 \mathrm{~cm})$ et celle des saumons $(70 \mathrm{~cm})$.

Le tunnel a été équipé d'un entonnement et placé en légère contre-pente (Fig. 3); l'angle d'inclinaison, de $10^{\circ}$ environ, place ainsi l'orifice amont "en pression" et élimine les risques de comptages erronés dus au passage de bulles provenant de la formation d'un vortex près de cet orifice. Le niveau d'eau doit être maintenu au minimum de 30 à $40 \mathrm{~cm}$ au-dessus de la partie supérieure du tunnel.

Une grille placée dans l'échancrure de la cloison oblige les poissons à traverser le tunnel, tout en autorisant l'écoulement du débit supplémentaire.

Signalons enfin que la chambre de la capture située immédiatement en amont est équipée d'un entonnement avec système de grille anti-retour. Compte tenu de ces équipements et du fort courant d'eau dans la passe vidangée entièrement à chaque contrôle, on pouvait raisonnablement penser que pratiquement tous les poissons ayant traversé le tunnel de comptage seraient ensuite capturés. 


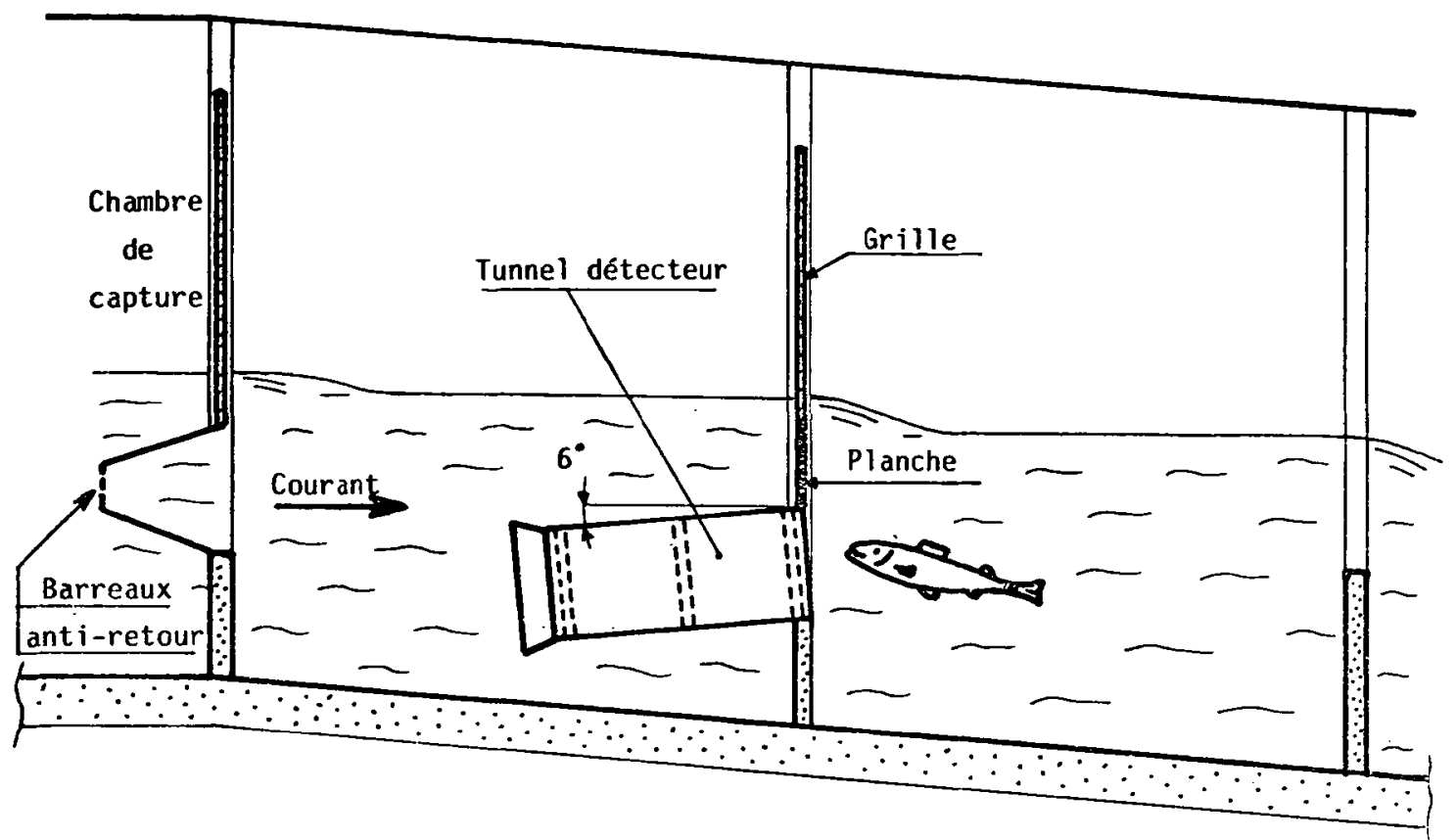

Figure 3 : Vue simplifiée de l'échelle à poissons et du tunnel détecteur.

Figure 3 : Sectional outline of the fish ladder and detector tunnel.

\subsection{4. - Les conditions électriques de fonctionnement}

Divers essais effectués en pisciculture, dans une eau dont la conductivité est identique à celle de la Nivelle, ont permis d'observer qu'une réaction de gêne apparente d'un saumon de $60 \mathrm{~cm}$ de longueur environ se manifeste lorsque la tension alternative d'alimentation du pont (VEam-VEav) est supérieure à $5 \mathrm{~V}$ crête. Afin de donner au compteur une sensibilité maximale, le voltage a donc été réglé à une valeur légèrement inférieure à ce seuil, soit $4 \mathrm{Vc}$. Dans ces conditions, ce même poisson est compté sur les compteurs $P$ et $G$ si le gain de l'amplificateur (par. 1.2.2.) est réglé sur la position 8 du vernier et si les tensions de référence des comparateurs $P$ et $G$ sont respectivement ajustées à 1.5 V et $2 \mathrm{~V}$. Une truite de $40 \mathrm{~cm}$ de longueur n'est, par contre, comptée que sur le compteur $P$. Ces réglages ont été adoptés pour une première série de tests en fonctionnement normal.

Les résistances inter-électrodes du détecteur implanté dans la passe à poissons ont été mesurées au pont de Kohlrausch, soit:

$R(E a m-E c)=R(E c-E a v)=R(E a m-E a v)=70$ ohms $=R(1)$ pour $\gamma e=215 \mu S / \mathrm{cm}$.

Le schéma électrique équivalent (simplifié) est donné à la figure 4, avec par exemple :

$R($ Eam $-E c)=\frac{R 1(\text { Rext. }+R 2)}{R 1+R 2+\text { Rext. }}$

Pour que l'égalité (1) soit possible, on doit avoir:

$\mathrm{R} 1=\mathrm{R} 2=$ Rext. $=\frac{3}{2} \mathrm{R}=105 \Omega$

On constate donc que la valeur de la résistance "externe" (Rext.) du tunnel est égale à la moitié de la valeur de la résistance "interne" (R1 + R2) et que, par conséquent, la puissance totale dissipée est 3 fois plus élevée que la puissance "utile" à la mesure. $P=0.23 W$.

Puissance totale dissipée : $P(W)=\frac{V c^{2}(V)}{R(\Omega)}$ avec $V c=V$ crête $=4 V$ et $R=70 \Omega$ soit, dans ce cas.
$23 \mathrm{~W}$.

Du fait de l'absence du courant secteur sur le site. l'appareil a été alimenté par une batterie d'accumulateurs supplémentaire de $24 \mathrm{~V}$ et $40 \mathrm{~A}$.h durant toute la période des essais. 


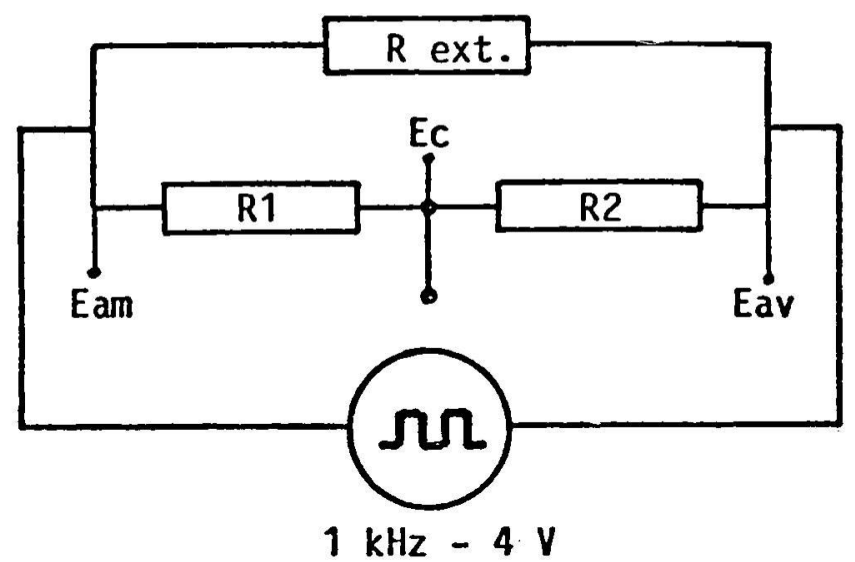

Figure 4 : Circuit équivalent simplifié du système d'électrodes.

Figure 4 : Simplified equivalent circuit of electrodes device.

\section{II - RÉSULTATS}

Les différentes espèces ayant emprunté la passe sont, dans l'optique compteur à résistivité, suffisamment similaires d'un point de vue morphologique pour que l'on puisse retenir uniquement la longueur comme seul paramètre allométrique dans l'analyse des résultats.

La passe a été mise en service le 9 mars 1985 et a fonctionné de manière continue jusqu'au 7 janvier 1986, excepté 7 jours de fermeture dus à des crues de la rivière. L'histogramme de fréquence des captures toutes tailles confondues est donné à la figure 5 .

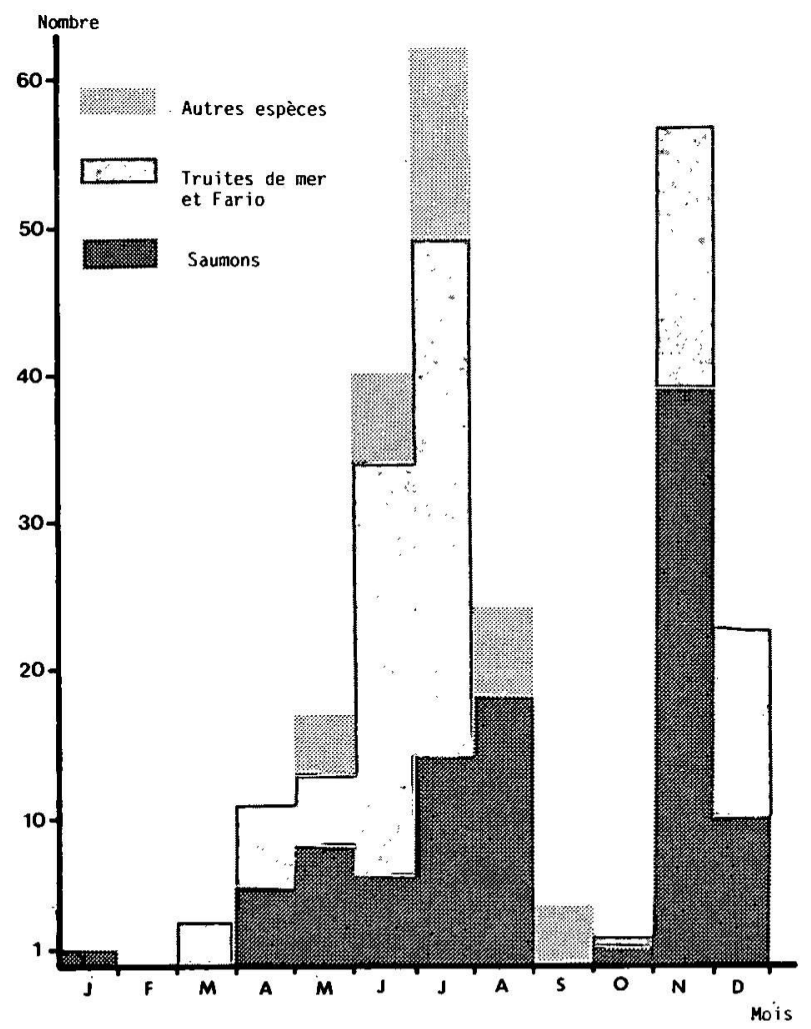

Figure 5 : Fréquence des captures à la passe à poissons d'Uxondoa du 9 mars 1985 au 7 janvier 1986.

Figure 5 : Trapped fish frequency distribution at Uxondoa fish pass, 9 March 1985 - 7 January 1986. 
Après une période de mise au point relativement longue compte tenu du faible nombre de passages, le compteur a été mis en service effectif et contrôlé régulièrement entre le 10 mai 1985 et le 7 janvier 1986. De cette période, il faut déduire 65 jours de non fonctionnement imputables aux crues, à des changements trop tardifs de la batterie et à une panne de l'appareil.

Le tableau 1 donne la répartition en classes de taille des poissons capturés pendant la période de fonctionnement considérée. Dans la ligne "truites", on a additionné les truites fario (17 au total) et les truites de mer et sous l'appellation "autres espèces"on a regroupé les aloses, les mulets et quelques spécimens de chevesnes, rotengles et perches.

Pendant cette même période, la somme des relevés journaliers des compteurs a donné : $P=174$ et $\mathbf{G}=127$, soit :

nombre de "petits" $=174-127=47=\mathrm{Np}$

nombre de "gros" $=127=\mathrm{Ng}$

De l'examen détaillé des captures et comptages journaliers qui, du fait de leur lourdeur, ne sont pas présentés dans cet article, il ressort également que:

- les poissons dont la longueur est inférieure a $30 \mathrm{~cm}$ ne sont pratiquement jamais comptés ;

- les poissons dont la longueur est comprise entre 30 et $40 \mathrm{~cm}$ sont comptés irrégulièrement;

- les poissons dont la longueur est comprise entre 40 et $50 \mathrm{~cm}$ sont parfois comptés sur les 2 compteurs, mais le plus souvent sur le compteur $P$ uniquement :

- les poissons dont la longueur est supérieure à $50 \mathrm{~cm}$ sont presque toujours comptés sur les 2 compteurs.

Si l'on élimine les animaux d'une longueur inférieure à $30 \mathrm{~cm}$, on peut exprimer le rapport: $\frac{\text { nombre total de poissons capturés }}{\text { nombre total de poissons comptés }}=\frac{136}{174}=0,78$, soit $78 \%(2)$

\begin{tabular}{|l|c|c||c|c|c|c|c|c|c|}
\hline $\begin{array}{l}\text { Longueur } \\
\text { à la fourche } \\
(\mathrm{cm})\end{array}$ & & 30 & 40 & 50 & 60 & 70 & 80 \\
\hline Saumons & 0 & 0 & 1 & 6 & 27 & 16 & 3 \\
\hline Truites & 14 & 42 & 13 & 5 & 3 & 0 & 0 \\
\hline Autres espèces & 5 & 15 & 4 & 1 & 0 & 0 & 0 \\
\hline Total & 19 & 57 & 18 & 12 & 30 & 16 & 3 \\
\hline
\end{tabular}

Tableau 1 : Répartition en classes de taille des poissons capturés à la passe d'Uxondoa pendant la période de comptage, 10 mai $1985-7$ janvier 1986.

Table 1 : Fish lengths composition of fish trapped at Uxondoa fish pass during the counting period, 10 May 1985 - 7 January 1986.

\section{III - DISCUSSION}

La précision du comptage exprimée par la relation (2) paraît satisfaisante, mais sans doute un peu flatteuse. En effet, l'examen des données journalières montre:

- qu'il existe quelques données aberrantes (non expliquées à ce jour),

- que la distribution des points obtenue en traçant le graphe de la figure 6 - nombre de poissons comptés en fonction des nombres de poissons capturés, toutes tailles supérieures à $30 \mathrm{~cm}$ confondues - n'est pas normale.

Au vu des résultats globaux, on pourrait tout d'abord penser que la séparation $P$ - $G$ intervient pour une taille inférieure à $40 \mathrm{~cm}$; l'intervalle $30-40 \mathrm{~cm}$ compte 57 spécimens $(\mathrm{Np}=47)$ et 79 
poissons ont une taille supérieure à $40 \mathrm{~cm}(\mathrm{Ng}=127)$. Mais si l'on se reporte une nouvelle fois aux résultats journaliers, on constate que cette déduction est erronée. Lorsqu on observe un passage isolé d'un poisson d'une longueur inférieure a $50 \mathrm{~cm}$, cas où il ne peut y avoir confusion avec d'autres passages, on s'aperçoit qu'il est, le plus souvent, comptabilisé sur le compteur $P$ uniquement. La limite entre $P$ et $G$ semble donc plutôt se situer aux environs de $50 \mathrm{~cm}$ et il paraît très difficile de la définir avec une incertitude inférieure à $\mp 5 \mathrm{~cm}$. Cette imprécision, que l'on retrouvera d'ailleurs dans la définition de la taille minimale, est en fait liée au principe même de la mesure et dépend de la forme et des dimensions du détecteur (SIMPSON, 1978). Le champ électrique est plus faible dans le centre du tunnel que près des électrodes, donc un poisson peut induire un signal plus ou moins intense suivant qu'il passe dans l'axe du tunnel ou près des parois. Pour les mêmes raisons, deux poissons de taille différente peuvent donner une information identique.

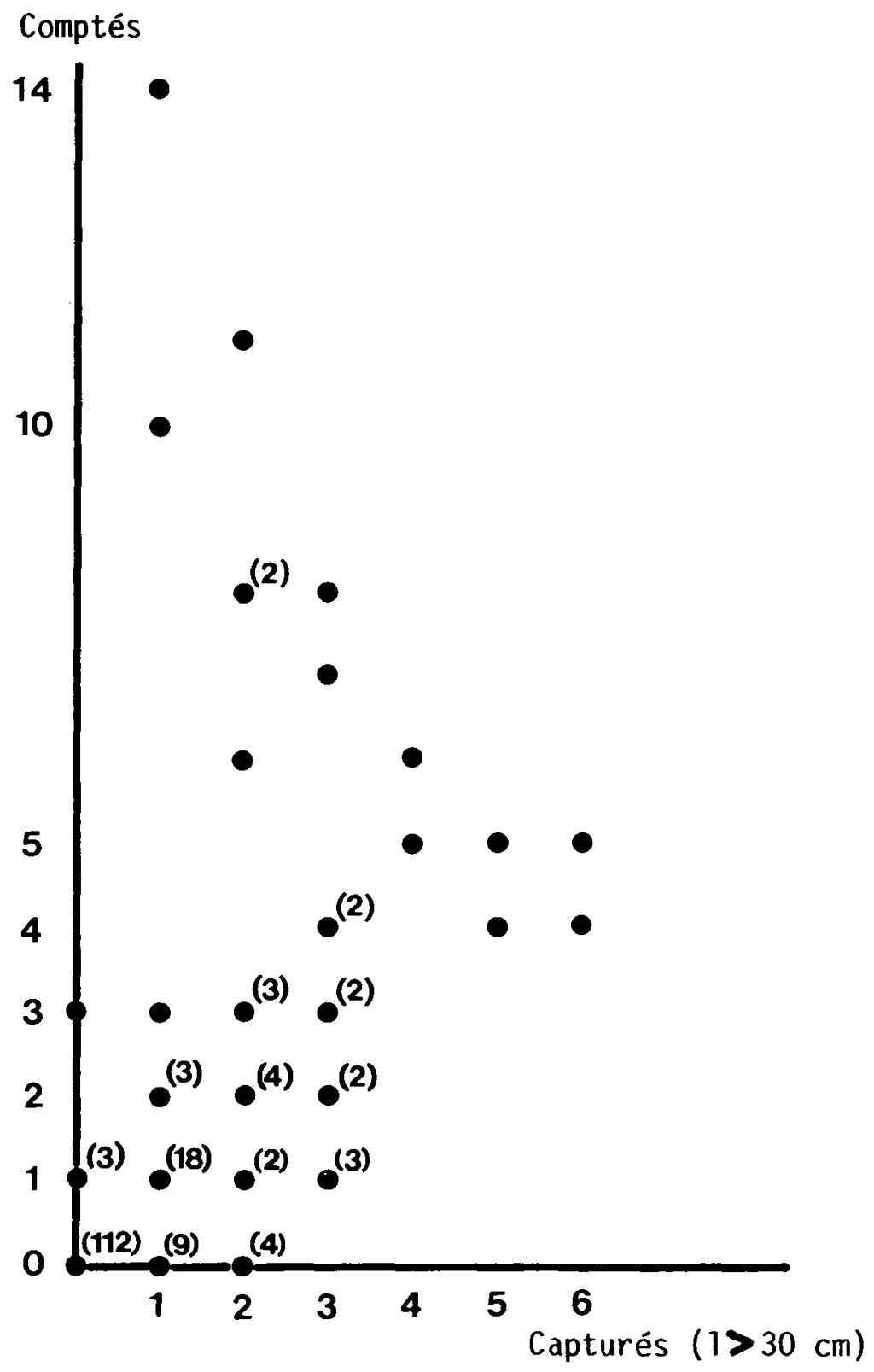

Figure 6 : Nombre de poissons comptés en fonction des nombres de poissons capturés (longueur supérieure à $30 \mathrm{~cm}$ ) pendant la période de fonctionnement du compteur. ( ) : nombre d'observations.

Figure 6 : Numbers of recorded fish in relation to the numbers of trapped fish (length superior to $30 \mathrm{~cm}$ ) during the working period of the counter. ( ): observations number. 
Si, malgré tout, on situe d'une façon relativement grossière la limite $P-G$ à $50 \mathrm{~cm}$, en éliminant toujours les animaux d'une longueur inférieure a $30 \mathrm{~cm}$, on constate que le nombre des poissons comptés est:

- inférieur au nombre réel $(\mathrm{Np}<75)$ pour $\mathrm{P}$,

- supérieur au nombre réel ( $\mathrm{Ng}>61$ ) pour $\mathrm{G}$.

Dans les deux cas, et sutout dans le premier, on peut penser que l'incertitude dont sont assorties les limites inférieure et supérieure est la principale cause de distorsion.

Dans le second cas, la différence est plus importante alors que la précision du compteur devrait, compte tenu du principe de la mesure, augmenter avec l'accroissement de la longueur. II est fort probable que la surestimation est surtout due au comportement des grands poissons dans le tunnel de comptage. Bien que les conditions hydrauliques existant dans la passe pouvaient laisser supposer, a priori, que les passages seraient francs et rapides, il a été observé que les saumons, en particulier, ne sont absolument pas gênés par la vitesse pourtant élevée du courant dans le tunnel ( $\mathrm{v}>1 \mathrm{~m} / \mathrm{s})$ et franchissent celui-ci sans effort apparent. Quelques comptages multiples, pour un seul spécimen capturé, semblent prouver que certains d'entre eux ne traversent le tunnel qu'après plusieurs hésitations ou mouvements de va-et-vient. Lorsque la passe est en fonctionnement normal, les retours vers l'aval sont également possibles mais plus improbables. Par contre, des observations récentes ont montré que quelques animaux, qui ont traversé le tunnel de comptage mais n'ont pas encore pénétré dans la chambre de capture au moment de la vidange, dévalent précipitamment lorsqu'ils perçoivent la baisse rapide de niveau. II s'avère donc que les précautions prises lors de la conception de l'appareil (par. 1.2.2.) et de l'installation du détecteur (par. 1.2.3.) ont été insuffisantes dans ce cas.

Pour pallier cet inconvénient, on peut faire un double comptage, sens amont et sens aval, et considérer que le nombre réel des remontées est la différence des deux relevés (STRUTHERS et STEWART, 1984; STRUTHERS et STEWART, 1985). Cette méthodologie peut dans certains cas paraître mieux adaptée, mais les deux exemples traités montrent que la précision n'est pas toujours supérieure. En effet, rappelons les résultats obtenus, dans des conditions comparables, au barrage de CLUNIE sur la rivière TUMMEL en Écosse:

- en $1983: \frac{\text { Nombre de saumons capturés }}{\text { Nombre de saumons comptés }}=\frac{176}{82}=2,14$

- en 1984, l'expérience a été renouvelée et on observe une amélioration spectaculaire de la précision avec:

$\frac{\text { Nombre de saumons capturés }}{\text { Nombre de saumons comptés }}=\frac{213}{191}=1,12$.

Les changements de conductivité de l'eau, consécutifs à de fortes pluies par exemple, ou le fait que deux poissons peuvent passer simultanément, sont également sources d'erreur. D'après les contrôles ponctuels de conductivité effectués et le faible nombre de poissons, il semble dans le cas présent que ces inconvénients soient de bien moindre importance que les précédents.

Depuis le remplacement d'un convertisseur de tension déficient, l'appareil a fait preuve d'une parfaite fiabilité électrique et fonctionne pratiquement depuis plus d'un an sans interruption.

Notons, enfin, que la sensibilité peut être accrue en agissant sur les réglages "gain" et "seuil $P^{\prime \prime}$ de l'unité électronique, ou en utilisant un tunnel de plus faible section. Par une légère modification technique, on peut également obtenir un comptage dans les deux sens.

\section{IV - CONCLUSION}

De cette première expérience de comptage automatique menée à Uxondoa on peut d'ores et déjà tirer divers enseignements.

En comparaison avec les résultats obtenus au Royaume-Uni en particulier avec ce type de compteur, il apparaît que la précision obtenue dans les conditions présentes d'utilisation est acceptable. On peut avoir une indication approximative mais raisonnable sur l'importance de la population ichtyologique effectuant des migrations ou des déplacements à un endroit déterminé, l'application la plus intéressante étant bien sûr le comptage des salmonidés aux barrages ou dans les échelles à poissons.

Utilisé comme outil de recherche ou de gestion dans l'aménagement des pêches, le compteur doit par contre présenter une meilleure précision; c'est dans ce sens qu'il est nécessaire d'améliorer l'installation et l'ensemble de l'appareillage.

En premier lieu, on cherchera une meilleure adaptation du tunnel détecteur aux conditions de mesure (site, taille, espèces). II faut en particulier créer des conditions principalement hydrauliques et visuelles, qui incitent l'animal à traverser le tube d'une manière franche et le dissuadent d'hésiter ou de revenir en arrière. 
L'effort doit également être porté sur les perfectionnements techniques de l'appareilélectronique: prise en compte de paramètres de mesures plus nombreux et analyse plus fine du signal au niveau de l'étage logique de l'unité de comptage. L'utilisation de la technologie des microprocesseurs, par exemple, offre des possibilités intéressantes.

Enfin, on peut penser que l'installation et le suivi de plusieurs compteurs dans des sites différents contribueront également à l'amélioration des performances. Avec des résultats multiples on peut envisager par exemple la détermination d'une courbe ou d'un facteur correctif conduisant à l'ajustement des résultats bruts.

Cette expérience de comptage sera poursuivie à Uxondoa en 1986. Quelques modifications, au niveau du tunnel notamment, seront testées. Nous espérons également qu'une surveillance fine et individuelle des passages (enregistrements des signaux ou contrôle vidéo) conduira à une connaissance comportementale plus approfondie et, par conséquent, à une meilleure interprétation des résultats.

\section{BIBLIOGRAPHIE}

BELL W.H., ARMSTRONG M.C., 1970. A photo-electric fish counter. Fish. Res. Board Can. Tech. Rep., $215,23 \mathrm{p}$.

BUSSELS R.B., 1978. Fish counting stations. Notes for guidance in their design and use. Department of the Environment. Londres, $97 \mathrm{p}$.

COUSENS N.B.F., THOMAS G.A., SWANN C.G., HEALEY M.C., 1982. A review of salmon escapement estimation techniques. Can. Tech. Rep. Fish. Aquat. Sci., 1108, 122 p.

DUMAS J., 1986. La population de saumons de la Nivelle. Bilan 1977-1985. Rapport interne INRA, Station d'Hydrobiologie, Saint-Pée-sur-Nivelle, 7 p. dact.

DUMAS J., CASAUBON J. Connaissance et restauration de la population de saumons atlantiques (Salmo salar L.) de la Nivelle (Pyrénées-Atlantiques). In THIBAULT M. et R. BILLARD (eds), La restauration des rivières à saumons. Colloque franco-québécois, mai-juin 1985, Bergerac. INRA Publs, Paris (sous presse).

LARINIER M., 1983. Guide pour la conception des dispositifs de franchissement des barrages pour les poissons migrateurs. Bull. Fr. Piscic., $n^{\circ}$ spécial (juillet), $39 \mathrm{p}$.

SHENTIAKOV V.A., 1959. Une méthode de détermination de la conductivité électrique du corps du poisson. Bull. Inst. Biol. Lacs Artificiels, 3, 45-46 (traduit du Russe).

SIMPSON D., 1978. Electrical resistivity fish counters. In Thorpe, J.E. (ed.), Rhythmic activity of fishes, 259-268, Academic Press, Londres.

STRUTHERS G., STEWART D., 1984. A report on the composition of the adult salmon stock of the Upper River Tummel, Scotland, and the evaluation of the accuracy of a closed-channel resistivity counter. ICES Doc. CM 1984/M : 20, 19 p. dact.

STRUTHERS G., STEWART D., 1985. The composition and migrations of the adult salmon stock in the Upper River Tummel, Scotland, in 1984, with observations on the accuracy of resistivity counter at two fish passes. ICES Doc. CM 1985/M: 14, 18 p. dact. 